\title{
Analysis of approaches to study identification in social media
}

\author{
Vera Orlova ${ }^{1, *}$, Vyacheslav Goiko ${ }^{2}$, Yulia Alexandrova ${ }^{2}$, Evgeny Petrov ${ }^{2}$ \\ ${ }^{1}$ Tomsk State University of Control Systems and Radioelectronics, Lenin Avenue, 40, 634050, Tomsk, \\ Russia \\ ${ }^{2}$ NI Tomsk State University, Russia
}

\begin{abstract}
The nature of the social influence of media on social processes, the production of virtual information practices, to study these processes currently actualizes the need to use modern new tools for collecting, processing and data analysis methods. The purpose of this work is to analyze the activity of university graduates in communities, their identification through the collection of data from social networks. Assessment of the activity of graduates in social networks was carried out by "downloading" messages and news from online university communities. For each message, activity labels ("likes", reposts, comments) were collected and graduates of these universities were identified (reconciliation with the register of graduates was carried out). The focus of the analysis is on identifying the actions of graduates - loyalty in the media space and the dissemination of information about the university community. The main methodological guideline was the approach within the framework of the microsociological paradigm, in particular, the idea of symbolic interactionism. The heuristic potential of using big data to analyze the activity of university graduates in communities allows us to expand our methodological arsenal and overcome the limitations of existing traditional methods of collection and analysis. The main research methods: interface programming, social network analysis of user interaction in social media, Web-crawling using a search engine, statistical data processing. Results: the main digital strategies of university graduates are characterized by the expansion of the audience, the promotion of content caused by the interest of users depending on the focus of the group. Four types of alumni communities have been distinguished: groups that identify with social development, with charity, with scientific research, and education. The high average value of the activity index belongs to charitable foundations, followed by the community of culture and science. The lowest average value of the activity index is recorded in educational communities.
\end{abstract}

\section{Introduction}

Social media is a mass media space where user identification markers, such as interests, preferences, moods, and integration vectors, are found. The theoretical basis for the study of

\footnotetext{
* Corresponding author: orlova_vv@mail.ru
} 
identification in social media is the work of foreign and domestic authors. The research is based on the paradigm of symbolic interactionism (C. Cooley, J. Mead, M. Kuhn), within the framework of which scientists studied social interaction and coordination of actions of individuals. The paradigm of symbolic interactionism is rooted in the understanding sociology of M. Weber, who pointed to the need to understand the environment of people in which they are. $[1,2,3,4]$.

For the first time, the essence of social networks was determined by G. Simmel (2002), then P. Bourdieu (2007) identified and analyzed the specifics and architecture of social networks. A special place in the theoretical foundation of this study is the works of D. Coleman (2001), F. Fukuyama (1995), who studied the issues of social capital as a special resource influencing the dynamism of social networks Among applied research, we note the works of S. Keisler, L. Sproul (2001), M. Castells (2009), who devoted their work to the study of various aspects of social networks [ five]. A significant contribution to the study of new possibilities of information communication technologies was made by Russian scientists: Ya.N. Zasursky (1999) [6], D.A. Gubanov, D.A. Novikov (2010, 2014) [7], Leshchenko (2011).

The use of digital technology products is becoming the norm in everyday life, therefore, it is necessary to develop tools for studying the impact and consequences on people's behavior, the structure of their value orientations, and behavioral strategies. Since the data is reproduced by the users themselves (posts, likes, photos, etc.), data in various fields medicine, education, criminology, etc. become available to government departments, so it became possible to analyze the "digital footprint" that remains from the actions of a large number of people ... Thus, the heuristic potential of using big online data makes it possible to expand the methodological arsenal and overcome the limitations of existing traditional methods of collection and analysis (eg Survey methods) [5,6,7,8].

The modern transforming society has not yet come to an established state, new structures and functions have not yet acquired their final form, and theories describing industrial society are already losing their attractiveness. However, it would be wrong to believe that the new society is some absolutely new phenomenon not related to the past, as well as theories that will most objectively describe and explain the new state of society and the transition to it, will have origins and rely on previous knowledge ... The development of sociological theory, like society itself, has causal relationships, the past is hidden in the new, and the past continues in the new. To understand the possibilities of the necessary sociological theory for a rapidly changing society towards digitalization, it is necessary to turn to past and existing sociological theories, concepts, and currents of sociological thought. This approach will make it possible to best guess the possibilities of forming digital sociological theories.

Contemporary sociological research already contains a discussion about digital sociology. The concept of "digital sociology" was first introduced in a scientific article by Wynn in 2009 (Wynn, 2009). Although the term "digital sociology" has not yet entered fully into scientific circulation, sociologists have been involved in research related to the Internet since its inception. First of all, sociologists were interested in the problems associated with online communities, cyberspace and cyber culture. These studies stimulated the emergence of various areas of sociological knowledge, which received roughly such names as "cybersociology", "sociology of the Internet", "sociology of online communities", "sociology of social media", "sociology of cyberculture", etc.

In general, the modern paradigm of the information society can be represented as a global process of production and widespread use of information as a public resource, based on the massive introduction of methods and means of collecting, processing, transmitting and storing information and causing profound changes in the progressive nature of socioeconomic, political and sociocultural structures in society, significantly affecting the level and quality of life of the population 
When analyzing the activity of university graduates in communities, big data play a significant role, which makes it possible to study information about human behavior in real time, who, where, with whom interacts, including the study of social "contamination". Such data allow observing online activity through social connections of patterns of attitudes, emotions (Johanna Gereke, Max Schaub, Delia Baldassarri [9].

In addition, big data differs from other information communication tools not only by its large volume, high accumulation rate (they are created here and now, their volume can increase every second), but also, according to Meta van der Linden, Marc Hooghe, Thomas de Vroome, by the variety of forms, high discreteness, flexibility, which allows you to add new information and expand the volume (Meta van der Linden, Marc Hooghe, Thomas de Vroome, 2017). [10].

We can formulate the thesis: big data cannot but influence the development of social science. One of these aspects of influence is the need for special competencies from the field of computer science to process raw data into the format that researchers need. These include the analysis of large arrays of text data, which is associated with linguistics. Since social networks are the source of data, network analysis and machine learning allow you to solve additional research problems. In addition, there is the possibility of online experiments [11].

To understand the identification of university graduates in social media, the ideas of phenomenological philosophy (E. Husserl, M. Merleau-Ponty, M. Scheler) are of particular importance [12]. In the works of these authors, the problem of identification (recognition) of a person is thought of through the analysis of the construction of living space, as a personal manifestation of the social world based on value preferences. In social sciences, the problem of identification and identity are similar terms in meaning. The specificity of the first is in identifying oneself with others, a group. The second term reflects the foundations, values and views of the individual on life. According to existential philosophy (S. Kierkegaard, J.-P. Sartre, A. Camus), the adoption of a fateful decision by a person leads to the formation of personal identity $[13,14,15]$.

Considers the problem of personal identification in a risk situation, connecting it with the problem of the ratio of Self and Other. The possibility of searching for grounds for the coexistence of social subjects and the situation of incorrect interpretation of the presented "cultural codes" in social reality allows us to say that social media can also be attributed to a risky environment (Sam J. Silva, Lindsay K. Barbieri, Andrea K. Thomer) [16].

Within the framework of our research, N. Baim's work "Personal Connections in the Digital Age" is of significant importance, where the author considers the problem of using mediated language and non-verbal behavior that the user uses to develop and maintain communities in social networks, including representatives of different universities, search new relationships, and to maintain relationships in everyday life.

Based on the concepts of phenomenology, the use of mediated language in building connections in social networks, the authors understand identification as a process of identifying oneself with a group in order to maintain relationships. Based on the foregoing regarding the object of research, our assumption is as follows: in social media (Vkontakte, Facebook), university graduates (as well as other groups), building a unique living space, strive to maintain connections with each other, unite into communities based on their own interests, preferences, which allows for identification, determination of the types of communities, their activity, digital strategies.

The main research approach in studying the identification of university graduates in social media and their digital strategies is within the microsociological paradigm, based on largescale data on human behavior, such as maintaining and developing links between graduates and universities; collecting information about alumni in order to determine the types of communities in which they participate. It is important for us to highlight the types of communities and form a social portrait of the graduate through the collection of data from 
social networks. With regard to our research, the method of natural language processing is used to identify agents and actors, in the case of using publicly available data.

With the active dissemination of information and information technology, which determined the nature of the IV technological order (which is currently being formed), great opportunities have appeared for a qualitatively new stage of economic growth. This circumstance will contribute to the transformation of business, education, healthcare, etc. Note that the characteristic difference between the IV industrial revolution and the previous ones lies in the synthesis of physical, digital and biological technologies.

The term "digital economy" is associated with the name of Nicholas Nigroponte, who in 1995 in his book described the understanding of the economy "being digital". There is currently no single definition of the digital economy. Let's take as a basis two definitions given by experts from the Australian Government and the World Bank:

1.A global network of economic and social activities that are supported by platforms such as the Internet and mobile and sensor networks" (Australian Government);

2."The system of economic, social and cultural relations based on the use of digital information and communication technologies (World Bank).

At the 2016 Davos Annual Meeting on Mastering the Fourth Industrial Revolution, it was noted that by 2020 the revolution will bring best practices in robotics, autonomous vehicles, artificial intelligence, advanced materials, biotechnology and genomics. These events will transform the environment in which we live and working conditions, including some jobs will disappear, the need for others will grow. Obviously, this factor must be taken into account today in order to keep up with the times. In addition, the problem for the employment of workers is indicated, what skills (skillset) of personnel are required in various industries and regions. According to experts, with the advent of new products, new technologies and new ways of working using digital technologies, creativity, critical thinking and emotional intelligence of workers can become one of the in-demand skills in order to reap the benefits of these changes.

The main trends characteristic of the IV industrial revolution, such as 3-D printing, robotics, synthetic biology, new nano- and biomaterials are interconnected, since they use the advantages for each other based on the developments of each of them. The scientific and educational complex of Tomsk is represented by leading universities (TSU, TUSUR, TPU, Siberian State Medical University), where training programs are implemented in these scientific areas. Thus, modern generations of robots are developing towards enhancing human-machine interaction. Many previously unresolved problems associated with heart and cancer diseases that have a genetic component are now being effectively developed at the Tomsk Research Center.

It is obvious that new, intensive changes in society, its individual components, caused by digitalization, began to be poorly perceived, described and explained by existing sociological theories. At the same time, the emergence and rapid spread of digital technologies has significantly increased the changes that have practically affected all spheres of people's life from individual household to public and state.

For research purposes, in 2018, data from 65 communities of higher education graduates were analyzed, total $n=1369732$. For each university, both official and related (faculties, structural divisions) pages in the social network Vkontakte were identified. Identification was carried out by means of a search by the name of the university, and links on official sites were also used. For the purpose of the study, data from 101 endowment funds (FCC) of 76 funds of educational institutions (of which 72 are universities) were analyzed. For each Fund, sites were identified, as well as pages in various social networks (Vkontakte, Facebook, Odnoklassniki, Twitter, Instagram, YouTube, Telegram). Identification was carried out by means of a search by the name of the Fund, and links on official sites were also used. 
To identify communities, we used the identification of linguistic markers for texts on the user's wall, his communities and subscriptions, on the basis of which interests were identified. Key tools for identifying and predicting behavior patterns:

- Natural Language Processing (linguistic analysis of natural language);

- Social Network Analysis (a study of social networks that considers social relations in terms of network theory);

- statistical data processing;

- machine learning algorithms.

To extract data from social networks, both standard methods of working with the API are used, and the development of their own methods based on Web-crawling, exploratory information retrieval, based on working with archival databases is carried out. Collection and storage of data is carried out using parallel file systems for online access technologies of distributed and parallel programming. Using the getSubscriptions method, you can get a list of IDs of communities that a user is subscribed to, and by these IDs you can already query information about the community itself (name, description, number of members, etc.). In addition, the API in the social network of the Russian-speaking segment includes methods for obtaining data from the community wall or from the user's wall, photos, the time when the user is online, and much more.

- The processing of the data obtained in accordance with the goals and objectives of the project is ensured by the use of the following tools: social network analysis, actor-network theory, graph visualization technologies, methods of mathematical statistics and machine learning. Models were trained using machine learning algorithms (Support Vector Classification, Stochastic Gradient Descen). The appropriate parameters for the models were selected and tested. A cross-validation was performed, which consists in evaluating the analytical model and its behavior on independent data. Algorithm for automatic classification of messages by sentiment and spheres of public life: this classifier is designed to mark up text content in 19 categories of social, economic and political spheres of public life. The algorithm is built on the basis of machine learning methods: as a result of numerical experiments, the gradient boosting and random forest models were tested, with various variations of the algorithm parameters. It is based on a decision tree model. Gradient boosting and random forest models generate sequential and random sets of decision trees, respectively. Based on the results of numerical studies, a gradient boosting algorithm from the LightGBM library was selected, which showed the best result for the corresponding problem. The manually labeled dataset will be split into two parts, training and test sets. The learning process of the algorithm was carried out on a training set, and the determination of accuracy on a test set. The accuracy of the algorithm is the proportion of correctly predicted categories or sentiments of the test sample messages.

- For the Analysis of the activity of alumni in the university communities, such networks as Facebook, Odnoklassniki, Twitter, Instagram, Youtube, Telegram were not used due to the restrictions of these networks on the collection of open data of user profiles. In addition, the content of the official communities of universities in such large networks as Vkontakte and Facebook is the same.

To analyze the relationship between university graduates, the method of social network analysis (SNA) was used. The main research methods are:

- Front-end programming of applications for uploading data from social networks.

- Web-crawling using a search engine.

- Statistical data processing.

- Social network analysis - social network analysis of user interaction in social media

- Classification and semantic clustering of texts of community posts

Social media can be characterized as networks with complex topologies. Note that a common property of many large networks is that the nodes of the networks follow the scale 
distribution of the power law. This function was recognized as a consequence of two general mechanisms: 1) networks are constantly expanding by adding new vertices, 2) new vertices are attached to participants that are already well connected. The model based on these two components reproduces observed stationary distributions without scale, which indicates that the development of large networks is governed by robust self-organizing phenomena that go beyond individual systems. The thematic content of the content of groups in the official communities is significantly different, as there are more posts containing various information, ranging from reference to entertaining.

Despite the great variety, one can distinguish the prevailing topics of posts in communities of different categories. Cultural communities have "theater" and "exhibitions"; at universities - universities / admission and holding conferences / events / lectures. Charitable communities - the largest number of posts were devoted to medical topics, in the official communities of research organizations, one can single out such popular topics as education and innovation. Communities of social development organizations are dominated by messages about entrepreneurship and financial literacy, as well as leadership.

The analysis of the content in the groups of the social network Facebook showed that the most active were users who were in the "charity" communities. The thematic analysis of the content of communities on the Facebook network showed heterogeneity in all categories, however, it is worth noting that information about organizations is in the lead in all, the share of such materials is from $90 \%$ to $35 \%$. In addition, in the stream of publications by education communities, as well as charitable Foundations, a large share is occupied by messages about upcoming events / events. Acknowledgments / gratitude is the largest in the flow of charitable Foundations. And expert reports are most represented in social development groups.

Analysis of content and its perception by subscribers of communities on Twitter showed a fairly low average activity index for all categories of groups. The highest average value belongs to charitable foundations -2.8 . This is followed by the community of culture -1.8 and science - 1.4. The lowest average value of the activity index was recorded in educational communities - 1.2. Analysis of digital strategies and thematic structure of content of groups of various types showed that, as in other sites, the largest number of materials is devoted to information about the organization - from $83 \%$ to $54 \%$ for all categories. Cultural communities have a significant proportion of posts about promoting events / events. In addition, an analysis of the activity of subscribers in the communities of parent groups of organizations (universities) was carried out. It should be noted that the dynamics of activity in all categories of communities was unstable; the most uniform activity can be called the activity in cultural communities.

The greatest activity was noted among the communities of educational organizations, the average value of which is 14.9. Charitable communities have an average activity value of 11.9, while communities on the topic of "culture" have the lowest - 4.5.

The thematic structure of charitable communities is dominated by publications on medical topics, including calls for collecting donations for treatment / surgery. In second place are posts with announcements / post-releases about events. The third most popular topic for posts is charity and volunteering. Note that in $2 \%$ of messages the Endowment Funds themselves were mentioned.

In the thematic content of social networking groups at cultural institutions, messages about training / education can be distinguished. Publications about opera / theater and concerts and performances became the second most popular topic in these communities. You can also highlight publications on the topic of "cinema".

Another very important aspect of identification is the characteristics of a person's quality of life. How to form a general concept of the quality of life? In English sociology, there is not even such a concept. They replaced it with "quality of working life". This term appeared in the United States in the 1960s. The theoretical stimulus comes from early European work 
and experimentation with a socio-technical systems approach. It is an organizational project to measure the well-being of employees and their participation in decision-making and, as a consequence, functional efficiency.

In Soviet science, there was only the concept of "quality of labor" as a degree of complexity, intensity, and severity. The newest Russian encyclopedic economic dictionary says nothing about this either. And only sociologists have suggested that the quality of life is a set of conditions and characteristics of life.And on this basis, or rather without any foundation, a system of indicators of the quality of life is being built. Referring to Adam Smith, they came to the conclusion that the quality of life depends solely on material wellbeing, which is expressed in gross product per capita. But in itself it is not optimal for reflecting the quality of life because:

- shows the scale of production and only indirectly reflects consumption and income, ignoring the degree of socio-economic inequality;

- does not take into account the harm to the environment from production activities, while the ecological state of the environment in which a person lives directly affects the quality of life.

A detailed criticism of the approach based on the use of GDP as a characteristic of the material situation of the population is presented in the Report of the Commission on Measuring Economic Performance and Social Progress, prepared by J. Stiglitz, A. Sen and J.-P. Fitoussi under the United Nations Development Program (UNDP) at the request of French President Nicolas Sarkozy in 2008-2010. ...Therefore, this commission proposed an extremely broad model, in which the first place was given to the indicator: the degree of freedom from poverty, social inequality, access to education, health care. Economic wellbeing in this model is a tool for achieving happiness.

It is interesting that the concept of happiness is not an invention of the above commission, but a plagiarism of the program of King Jigme Wanchguk of Bhutan, who already in 1971 replaced the GDP indicator with GNS - gross national happiness, with its indicators: spiritual, physical, social and environmental health of citizens, active development, fairness of social processes, preservation of cultural heritage and the environment. For 34 years in Bhutan, this model has been implemented in practice, and the king entered the list of 100 people who shape our world, and since 2013 the UN has officially approved this indicator. It includes GDP per capita, life expectancy, civil liberties, corruption, generosity and generosity of citizens. In 2018, according to this indicator, the list of the 10 happiest citizens included: 1. Finland -7.632; Norway - 7.594; Denmark - 7.555; Iceland - 7.495; Switzerland - 7.487; Netherlands - 7.441; Canada - 7.328; New Zealand - 7.324; Sweden -7.314; Australia - 7.272. Russia with an index of 5.810 is on the 59th line of the rating between Northern Cyprus and Kazakhstan. (in 2015 - 64th place). The most unfortunate (2.905) were residents of Burundi - 156 last.

Thus, the concepts for assessing the quality of human life presented above are necessarily supplemented by sociological research. But the main difficulty is primarily associated with the fact that for almost a century, the methodology of research has been the theory of Durkheim - a person is a rationally thinking being, his brain reflects the objective world, which is rationally constructed by the brain in ideal models. All sociological research was carried out on this basis until the 21 st century. Of course, in the industrial era, a rigid class society, industrial sociology was a priority. Thousands of works have been published on the topic "Man and His Work". However, it turned out that the primary thing in the knowledge of the world was, is and will be emotion, an emotional assessment of the reflected subjective world. In other words, people construct the world on the basis of inherent genetic programs through smells, color, sound, etc. Ch. Kindleberger and Aliber proved that the cause of all 48 economic crises that have occurred over the past 300 years was the genetic program "greed and fear". For Russia, the clearest example is the Mavrodi pyramid. 
In 2017, the American economist R. Thaler received the Nobel Prize for discovering the primacy of emotions in the life of society. Scientific disciplines were born: the economics of emotions, neuromarketing, neurosociology as modern innovative projects for the analysis of human behavior. Alfa-Bank is the first organization in Russia where a hidden camera determines the level of satisfaction with the service based on the emotions on the client's face. This once again confirms Tarde's position that the social (our assessments, actions, etc.) is the result of the decomposition of the imaginary (existential). Thus, in modern conditions, the integral indicator of the quality of life is a system of objective and subjective indicators: GDP per capita, the degree of living comfort (housing, infrastructure, balanced nutrition, ecological state of the environment, accessibility and quality of education and health care, etc.) that passed through the filter of emotional assessments focused on the concept of happiness. It is by this methodology that a sociological survey in 2008 was conducted in the Russian Federation within the framework of the project "Social changes in modern Russia: measurement methodology and social practices". As an integral indicator of the quality of life, the authors chose social well-being as the unity of the objective and the subjective. To the first they attributed the material situation of people, health status, safety, medical, trade, transport services, housing and communal services, etc. The second is the subjective perception of the above areas of life. It turned out that in almost all indicators, except for trade, life in a planned distribution economy is estimated at an order of magnitude higher than in the best year of 2008, a market one, or more precisely a market without institutions. The basic indicators of social well-being: social dependence, confidence in the future, benevolent, collectivist attitudes were replaced by their antipodes. In the USSR, regardless of nationality, professional status, everyone was brothers, now everyone is enemies. Subjective assessments correlate well with objective indicators of the quality of life in the Russian Federation according to international ratings.

According to the human development index (life expectancy, the level of literacy of the country's population, the standard of living estimated through GNI per capita at purchasing power parity) - 50th place out of 188 countries. Malaysia has overtaken us in life expectancy and GDP per capita. The economy of simple things has been lost .. There are over 20 million people below the poverty line. and only by 2024 , according to the very optimistic forecast of A. Kudrin, we are going to reduce poverty by $25 \%$, the poverty of families with children by $40 \%$ (Kudrin A. Russia 24). But such rosy forecasts have already been made. 2003-2010 planned to increase GDP by $200 \%$, but in fact $-25 \%$. Since May 1,2018 , the minimum wage is equal to the subsistence minimum of 11,163 rubles. (-13\% tax). For the G20 countries, the minimum wage today is in the following amounts: Germany - 106,370 rubles, France 83,580 rubles, China - 31,700, Turkey - 29,160, South Africa - 11400, Brazil - 10780, India - 4060 rubles. ... And it turns out that the objective parameters of the quality of life in the Russian Federation have decreased in comparison with 1980 many times, and the subjective ones give a "rosy" picture of the current state. Why "pink" memories? Because "when information is extracted from the depths of our brain, it is rewritten again. And during extraction and rewriting, it can mutate in an incredible way, so that our memories are also transformed. And the person continues to think that this is the true information reflecting what is happening ... Both poles of memory, the cellular biological and cultural humanistic, are still little studied in interaction. " ...

Thus, studying the problem of the quality of life, we see how mobile and fluid the model itself, its indicators, depending on age, gender, mentality, ethnicity, are. So, for example, the basic indicator for a pensioner will consist of the characteristics of the state of consumer services: health protection, the level of pension, social security, psychological comfort in dealing with children, grandchildren, and the quality of food. For a working person - the quality of work, family life, leisure, etc. However, for a Mongol and a Frenchman, and a pensioner and a working person, this list also includes different contents. But in general, the 
Eastern type of personality with an inward-directed mentality (introvert) has a different model of life than the Western - extroverted one.

Entering the university is the main goal of a person after graduating from school. And the most important thing is to feel safe when entering a university. The solution to the safety problem is to ensure comfortable conditions for a person, in his protection and protection of his environment (urban, residential, industrial) from the effects of harmful factors that exceed the normatively permissible levels. It can be said that the task of ensuring the safety of a person's residence both in the city and on the campus is not to eliminate existing dangers, but to reduce the potential level of hazards and reduce the consequences of their actions. Therefore, further we will consider the campus (campus) in terms of security, comfort and living conditions, the degree of infrastructure development.

When choosing a campus compositional solution, the designer is faced with the task of creating a holistic space-planning composition. One of the main compositional techniques in the design of university campuses is the organization of a single composition of the complex, the generalization of form and space. He must also comply with the principles of aesthetics. The image of the building should be memorable and attractive, but at the same time laconic and balanced. Modern technologies provide great opportunities for the implementation of architectural designs of any complexity. Approaching the choice of color, it is worth abandoning severity and gloom, but also so that there are not too many bright colors. Previously, the campus was a maximum of comfortable conditions for students, especially for nonresidents. On the territory there was everything that a student needed, a library, a firstaid post, a reading room, and, of course, places of leisure. But time passed, the development of a generation went on, students changed and changed with them the place where they are during their studies at the university. Developed and expanded their campuses of a new level with a developed infrastructure, a new security system, adding and expanding the boundaries of the possible. It is very important for the person to feel safe and comfortable.

What is a campus (campus), what are the forms and their types? Typically, the layout of the campus is a rectangle where everything necessary for the educational process, comfortable living, organization of the cultural and leisure component of students should be. That is why the creation of such campuses should be equipped not only for the comfortable living of students, but for their successful learning. All university campuses have a developed infrastructure and an unusual architectural and construction image. The design idea mainly reflects the idea of a comfortable and comfortable home for students and teachers. The allocation of personal space for each student, which will reflect his interests and contribute to the successful development of the personality, becomes fundamental. As a rule, on the territories of such student campuses, university events, sports competitions between universities, faculties, students are rewarded for their achievements.

In total, there are three main types of university complexes:

- urban complexes of a distributed type;

- urban local complexes of integrated (campus) type;

- suburban (suburban) university campuses.

The first type: urban distribution complexes. At its core, it is, as a rule, not a campus, but a complex of university buildings and facilities that are scattered throughout the city. The vast majority of Russian higher education institutions have just this type of campus.

The second type: urban local complexes of the integrated type. They are high-density campuses located in urban areas. The isolation of the territory of such a complex, as in the first case, creates problems with the search for territories for further development and construction of new structures.

The third type: Suburban university campuses are the most promising and efficient. They are usually located outside the city and have significant advantages over the first two types of campuses. When located on the periphery in the immediate vicinity of the park zone, the 
possibilities of organizing a university complex are most widely disclosed, since here it is much easier to solve the problem of allocating a territory for construction.

However, it cannot be said that the first and second types of university complexes are unsuitable for scientific activity. There are cases when the typology of the placement of such complexes is very vague.

It is important to pay attention to the design features of university campuses: cluster structure during design; $\bullet$ organization of pedestrian accessibility of all structures of the complex; availability of territory for the possibility of further development and expansion; organization of flexible internal space, allowing redevelopment; irregular placement of buildings on campus; allocation of recreational space in the center of the campus; location of the campus in a picturesque landscape environment; high level of infrastructure development in the residential sector.

Finally, consider the campus in terms of quantitative parameters. And they are:

1. Microcampus in an urban environment. It assumes the concentration of all the minimum necessary functions of the complex in one object (International Business School in Skolkovo, Moscow).

2. Minicampus - this type is typical for classical universities,new universities (one university - up to 2000-5000 students).

3. Classic historic campus - with a system of malls and colleges. Oxford is a typical example. The college is a historical spatial scheme: the center of the composition was a quadrangular courtyard, usually square or rectangular, around which all functional volumes were formed.

4. Macrocampus - with high building density and complex structure, usually reconstructed and rebuilt many times.

5. Megacampus. This type consists of several universities (from 2 up to 10) with general social, engineering and transport infrastructure -up to 220,000 people (Megacampus in Guangzhou). As a rule, designing a student campus is a very important task, since it must reflect and collect the whole complex of composition that will reflect the individuality of the university.

Therefore, from all that has been said, we can conclude that the campus is a multifunctional complex that can become a center of scientific and cultural attraction. In the process of designing a campus, it is necessary to solve a number of important problems: urban planning, planning, functional, compositional, social, economic, environmental, structural, engineering and technical, etc.

\section{Conclusions}

Social media presents a wide range of methods for collecting public information about users. Since the content of the official communities of universities $(72,2018)$ in such large networks as Vkontakte and Facebook coincides, the identification of the analysis of strategies was carried out by searching by the name of the university, links on the official sites. The data of the networks Facebook, Odnoklassniki, Twitter, Instagram, Youtube, Telegram were not used due to the restrictions of these networks on the collection of open data of user profiles, which were necessary to analyze the activity of graduates.

Identification in the space of modern social media is realized as the reproduction of a unique living space by the subject based on knowledge about himself, his own interests, and preferences. Four types of communities of university graduates, identifying themselves with a group of social development, communities engaged in charity, science and culture, and education have been identified. It was determined that the main digital strategies of university graduates are characterized by the expansion of the audience, the promotion of content caused by the interest of users, depending on the direction of the group. The highest average value 
of the activity index belongs to charitable foundations, followed by the communities of culture and science.

The lowest average value of the activity index was recorded in educational communities. In the thematic content of groups of social networks of cultural institutions, one can single out messages about training / education, publications about opera / theater, on the topic of "cinema". In the group of alumni belonging to the category "education", posts with events are in the lead; a significant proportion of publications are devoted to posts about work / internships / projects and conferences. They also had a higher index than other categories, in addition, we note that most of the posts were about scholarships, programs and events, which allows us to conclude that it is this information that causes the greatest response from users.

\section{Acnowledgements}

Supported by RFBR 20-011-31154 opn.

\section{References}

1. K. Rost, L. Stahel, B.S. Frey, Digital Social Norm Enforcement: Online Firestorms in Social Media (2016) https://doi.org/10.1371/journal.pone.0155923

2. A. Ryan Rico, The University of Texas at Austin Fans of Columbine shooters Eric Harris and Dylan Klebold DOI: https://doi.org/10.3983/twc.2015.0671

3. E. Ferrara, Z. Yang, Measuring Emotional Contagion in Social Media (2015) https://doi.org/10.1371/journal.pone.0142390

4. K. Samson, Trust as a mechanism of system justification (2018) https://doi.org/10.1371/journal.pone.0205566

5. D. Antonakaki, D. Spiliotopoulos, C.V. Samaras, Social media analysis during political turbulence (2017) https://doi.org/10.1371/journal.pone.0186836

6. K. Okamura, S. Yamada, Adaptive trust calibration for human-AI col-laboration (2020) https://doi.org/10.1371/ journal.pone. 0229132

7. P.R. Ward, L. Mamerow, S.B. Meyer, Interpersonal Trust across Six Asia-Pacific Countries: Testing and Extending the 'High Trust Society' and 'Low Trust Society' Theory (2014) https://doi.org/ 10.1371/ journal. pone.0095555

8. T. Haesevoets, C. Reinders Folmer, A.Van Hiel, Is Trust for Sale? The Effe-ctiveness of Financial Compensation for Repairing Competence- versus Integrity-Based Trust Violations (2015) https://doi.org/ 10. 1371/journal.pone.0145952

9. J. Gereke, M. Schaub, D. Baldassarri, Ethnic diversity, poverty and social trust in Germany: Evidence from a behavioral measure of trust (2018) https://doi.org/10.1371/journal.pone.0199834

10. M. van der Linden, M. Hooghe, T. de Vroome, Extending trust to im-migrants: Generalized trust, cross-group friendship and anti-immigrant sentiments in 21 European societies (2017) https://doi.org/10.1371/journal.pone. 0177369

11. G. Bente, T. Dratsch, K. Kaspar, PLOS ONE 9(8), e107075 (2014) https://doi.org/10.1371/journal.pone.0107075

12. L.M. PytlikZillig, C.D. Kimbrough, E. Shockley, A longitudinal and experimental study of the impact of knowledge on the bases of institutional trust (2017) https://doi.org/10.1371/journal.pone.0175387 
13. D. McFarland, A. Goldberg, K. Lewis, American Sociologist 47(1), 12-35 (2015) https://www.semanticscholar.org/paper/Sociology-in-the-Era-of-Big-Data\%3A-TheAscent-of-McFarland-Lewis/dd1d3d4ce2df49c52649d3 b2781446d511892e14

14. Kazuo Okamura, Seiji Yamada, PLOS ONE 15(2), e0229132 (2020) https://doi.org/10.1371/journal.pone.0229132

15. F. Sabatini, F. Sarracino, Online Social Networks and Trust Social Indicators Research 4, 1-32 (2018) http://www.socialcapitalgateway.org

16. J.A. Hamm, C. Smidt, R.C. Mayer, Understanding the psycholo-gical nature and mechanisms of political trust (2019) https://doi.org /10.1371/journal.pone.0215835

17. S.J. Silva, L.K. Barbieri, A.K. Thomer, Observing vegetation phenology through social media (2018) https://doi.org/10.1371/journal.pone.0197325 